\title{
Thoracic spinal subdural hematoma complicating anterior cervical discectomy and fusion: case report
}

\author{
Nicole M. Protzman, MS, ${ }^{1}$ Jennifer Kapun, FNP, DNP, ${ }^{2}$ and Christopher Wagener, MD² \\ ${ }^{1}$ Clinical Education and Research, Coordinated Health, Allentown; and ${ }^{2}$ Orthopedics, Coordinated Health, Bethlehem, \\ Pennsylvania
}

\begin{abstract}
A spinal subdural hematoma is a rare clinical entity with considerable consequences without prompt diagnosis and treatment. Throughout the literature, there are limited accounts of spinal subdural hematoma formation following spinal surgery. This report is the first to describe the formation of a spinal subdural hematoma in the thoracic spine following surgery at the cervical level. A 53-year-old woman developed significant paraparesis several hours after anterior cervical discectomy and fusion of C5-6. Expeditious return to operating room for anterior cervical revision decompression was performed, and the epidural hematoma was evacuated without difficulty. Postoperative imaging demonstrated a subdural hematoma confined to the thoracic level, and the patient was returned to the operating room for a third surgical procedure. Decompression of T1-3, with evacuation of the subdural hematoma was performed. Postprocedure, the patient's sensory and motor deficits were restored, and, with rehabilitation, the patient gained functional mobility. Spinal subdural hematomas should be considered as a rare but potential complication of cervical discectomy and fusion. With early diagnosis and treatment, favorable outcomes may be achieved.
\end{abstract}

http://thejns.org/doi/abs/10.3171/2015.5.SPINE141191

KEY WORDS diagnostic imaging; spinal subdural hematoma; spinal cord compression; surgery; cervical; thoracic

\begin{abstract}
A LTHOUGH spinal subdural hematomas are a recognized clinical entity, they are a rare phenomenon. Prompt diagnosis and treatment are essential in preventing irreversible damage and permanent deficits. ${ }^{8}$ Spinal subdural hematomas are commonly associated with bleeding disorders and anticoagulation therapy, ${ }^{1,3,12}$ but they have also been reported following trauma and iatrogenic procedures, such as diagnostic lumbar puncture, acupuncture, and spinal anesthesia. . $^{1,3,4,7,8,10,12,14}$ Throughout the literature, few reports have described the occurrence of a spinal subdural hematoma following spinal surgery. $5,6,13$ To our knowledge, no study has detailed the onset of a spinal subdural hematoma at the thoracic level following surgery on the cervical spine. The present report describes a case in which a patient underwent an anterior cervical discectomy and fusion of C5-6 and postoperatively developed a thoracic subdural hematoma.
\end{abstract}

\section{Case Report}

History and Examination

A 53-year-old woman presented with neck and left upper-extremity radiculopathy, radiating to the thumb. Physical examination revealed left biceps muscle weakness. The patient had undergone repair of the left anterior labrum 3 months earlier and had been in postoperative physical therapy. She continued to have complaints of left upper-extremity weakness with new cervical neck pain and altered sensation to the left upper extremity. The patient underwent cervical spine MRI and was referred for surgical evaluation. The MRI study from December 2012 was consistent with multilevel degenerative spondylotic changes in the cervical spine with congenital narrowing of the canal, due to congenitally shortened pedicles. There was moderately severe central canal stenosis at C5-6 (Fig. 1), moderate 
to moderately severe bilateral neural foraminal stenosis with indentation of the C-6 nerve root, minimal to moderate right neural foraminal stenosis at $\mathrm{C} 4-5$ with possible abutment of the right C-5 nerve root, and minimal bilateral neural foraminal stenosis at C3-4.

Collectively, the patient's history, physical examination, and imaging studies suggested symptoms of C5-6 pathology. The risks, options, and benefits of surgical intervention were discussed in detail. The alternative of continued observation was also discussed. The patient elected to undergo anterior cervical discectomy and fusion of C5-6.

\section{Operative Course}

The patient underwent anterior cervical discectomy and fusion of C5-6. Intraoperatively, the C5-6 interspace was identified, marked, and confirmed with intraoperative imaging. The surgeon noted significant compression of the thecal sac and exiting nerve roots, secondary to degenerative change. Following gentle distraction, a complete discectomy of C5-6 was performed. The endplates were made relatively parallel. Decompression was continued through the posterior longitudinal ligament. Bilateral foraminotomies were performed until a nerve hook could easily be passed into the foramen. A polyetheretherketone biomechanical spacer was packed with local bone that was obtained from the decompression and impacted into place. A blunt nerve hook was placed around the posterior aspect of the graft to confirm its fixed position and clearance into the canal. The plate and screws were placed on the anterior aspect of the cervical spine. The screws were tightened to industry specifications, including a locking mechanism. The area was thoroughly irrigated, and the operative field was inspected for bleeding. No bleeding was noted. Surgery was uncomplicated with no unusual findings and minimal blood loss. No change in neurophysiological monitoring was noted. At the completion of the surgical procedure, the patient was able to move all extremities upon command.

\section{Postoperative Complication}

The immediate postoperative course was complicated by severe hypertension (systolic blood pressure 180-200 $\mathrm{mm} \mathrm{Hg}$ ), which was treated with intravenous medication. The patient was transferred to the floor with grossly normal neurological examination findings. During this period, the patient had complaints of upper thoracic pain and continued to have intermittent elevated blood pressure readings. Approximately 4 hours after surgery, the patient developed a substantial neurological deficit with absent motor and sensory nerve function in the bilateral lower extremities and giveaway weakness in the bilateral upper extremities. Upon examination, the patient demonstrated bilateral foot drop with an inability to dorsiflex, plantar flex, or wiggle her toes. Decreased sensation with subjective numbness and tingling was noted from her thighs to her toes and in the hips, pelvis, and abdominal region. The patient also demonstrated moderately decreased hand grip bilaterally.

\section{Second Operation}

The patient was urgently transferred to the operating
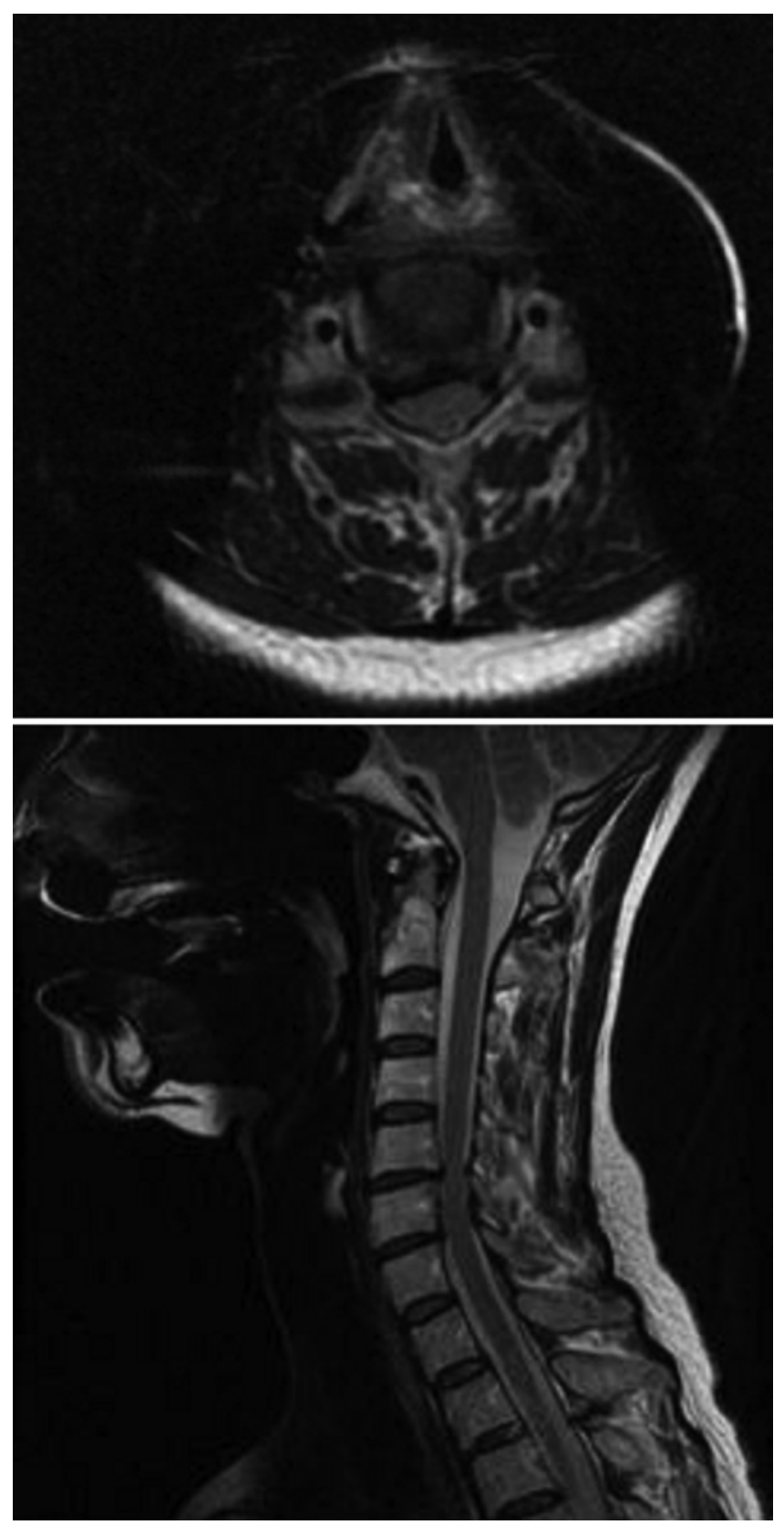

FIG. 1. Preoperative MR images. Axial (upper) and sagittal (lower) T2weighted images demonstrate moderate to moderately severe central canal stenosis at the C5-6 level.

room where an anterior cervical revision decompression and removal of instrumentation was performed. The postoperative hematoma was noted at the level of the thecal sac at C5-6. It was evacuated without difficulty, and the instrumentation and graft were reinserted. No complications were encountered intraoperatively. Following the procedure, the patient remained intubated and was taken to the MRI suite to evaluate the decompression.

\section{Radiographic Findings}

Cervical imaging was performed, which showed a capacious canal at the C5-6 level with no evidence of cord compression (Fig. 2). However, in the periphery of the cer- 


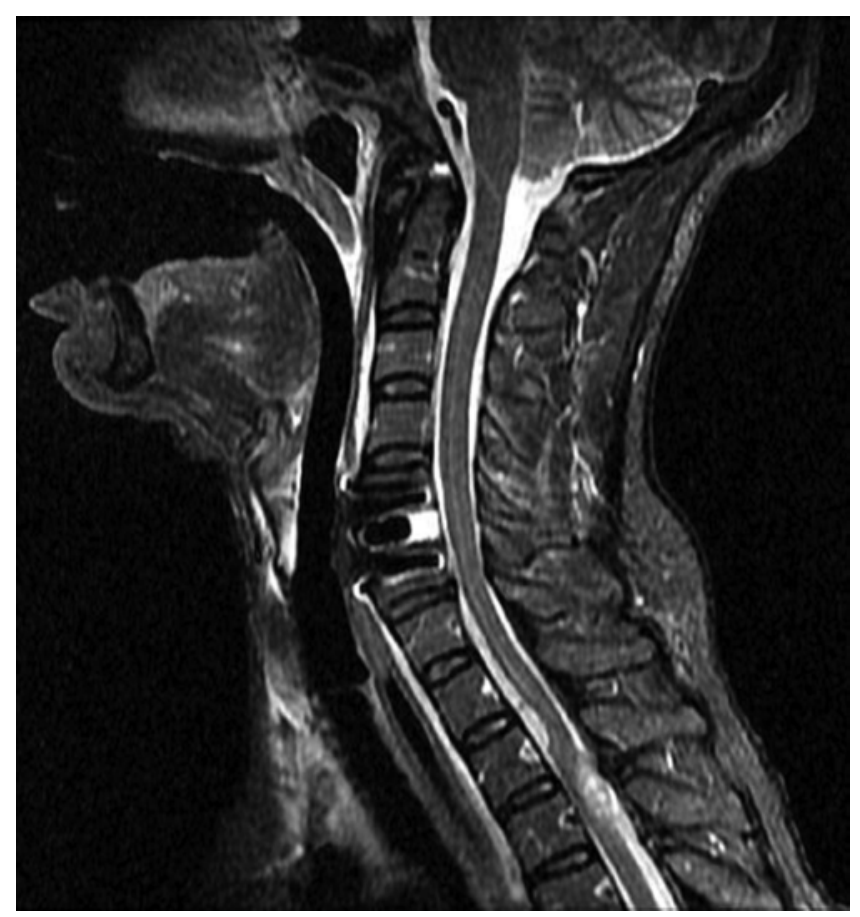

FIG. 2. Sagittal cervical T2-weighted MR image obtained immediately after epidural decompression, demonstrating the peripheral T2-3 subdural lesions.

vical images, there were 2 areas of spinal cord compression. There was no evidence of edema in the cord. Postcontrast dedicated imaging of the thoracic spine was performed. A subdural hematoma was noted at T2-3, causing severe compression of the thecal sac (Fig. 3). Given the significant sensory and motor deficits observed postoperatively, the surgeon elected for urgent surgical decompression. The patient was taken immediately to the operating room.

\section{Third Operation}

A laminectomy of T-1, T-2, and T-3, including partial facetectomies, was performed. A linear durotomy was made, and a hematoma was noted within the subdural space. It was evacuated at both levels. Following evacuation of the thecal sac, the surgeon noted evidence of a pulsatile flow within the dura, which was not appreciated prior to the decompression. No complications were encountered intraoperatively. The patient was subsequently taken to the neurosurgical intensive care unit in a stable condition.

\section{Postoperative Course}

Over the course of 48 hours, the patient demonstrated substantial improvement in neurological function. The patient was noted to be awake and alert and oriented to person, place, and time with clear speech. Cranial nerves II though XII were grossly intact, although the neck was not tested because of the cervical collar. Nonspecific mild weakness was documented in her bilateral upper extremities. Bilateral lower-extremity muscle strength was $5 / 5$ with the exception of $4 / 5$ in the right quadriceps muscle. Sensation was grossly intact.

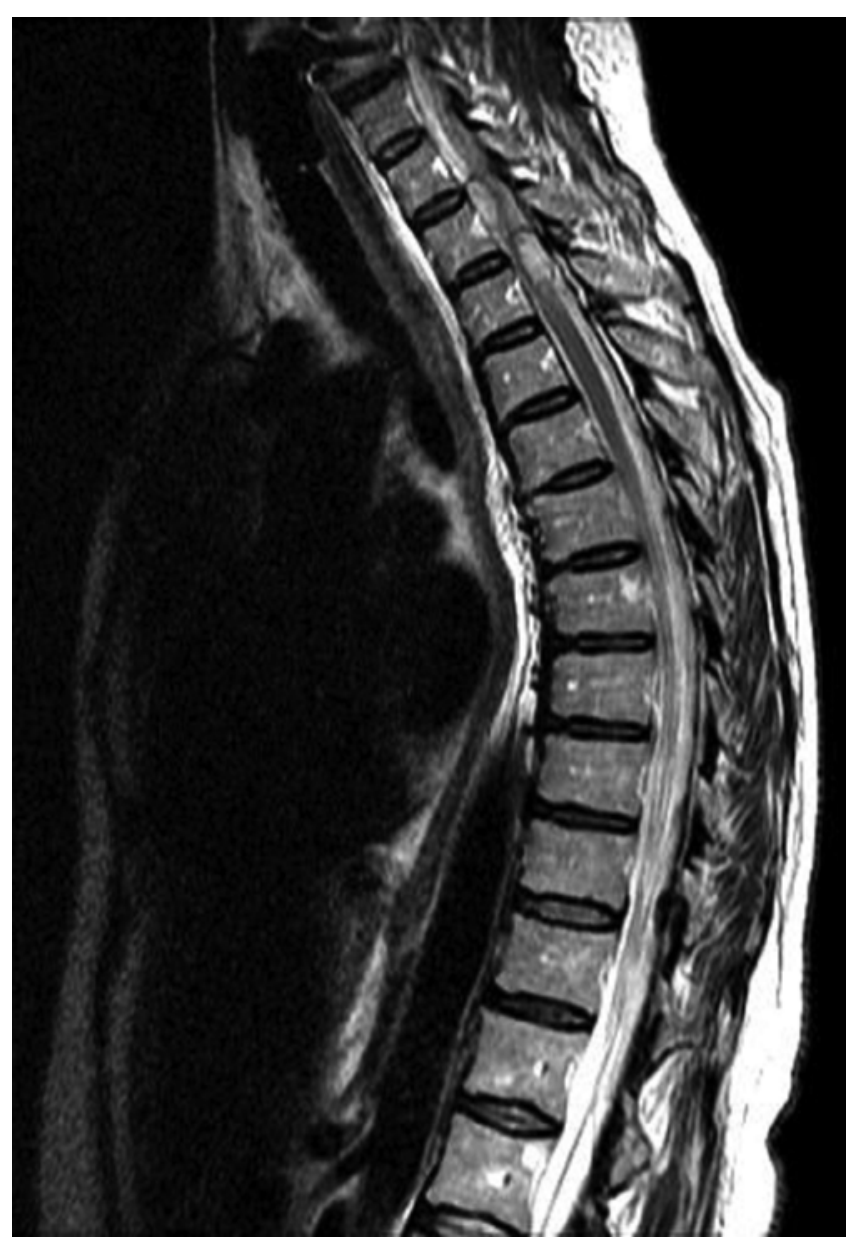

FIG. 3. Dedicated thoracic MR image obtained after the second procedure, demonstrating an epidural and possible subdural hematoma at T-2 and T-3, causing compression of the thecal sac.

A hematology/oncology consultation was obtained to rule out an underlying bleeding disorder that may have contributed to the thoracic hematoma. The patient had a number of past surgical stressors without any significant bleeding complications. There was no family history, which strongly argued against a congenital defect. Although the patient was hypertensive postoperatively with systolic blood pressure in the range of $180-200 \mathrm{~mm} \mathrm{Hg}$ systolic, the consultant noted that it is uncommon for aneurysms to manifest in this fashion. Vasculitis was considered, but the patient showed no systemic manifestations. A vertebral arteriogram was performed, which ruled out any evidence of spinal dural arteriovenous fistula or malformation. In summary, the consultant concluded that there was no obvious evidence of bleeding diathesis, especially in light of a completely negative bleeding history and family history. After extensive rehabilitation, the patient demonstrated functional ambulation.

The patient was most recently seen in July 2014 for follow-up. The incision was well healed. No masses were palpable in the cervical region, and no palpable lymphadenopathy was found in the neck. The patient's gait was steady with a rolling walker, but she requires standby assistance for toe walk and heel walk. The patient's reflexes 
were $2+$ and symmetric, except for a brisk reflex in the right patella. The patient had absent ankle clonus bilaterally. The extremities were intact to light touch in all dermatomes, except the right anterior thigh. The patient is being followed up with serial neurological examinations.

\section{Discussion}

A spinal subdural hematoma is a rare condition with significant consequences if diagnosis and treatment are not expedited. ${ }^{3,12}$ Cases of permanent paraplegia and persistent neurological deficits have been attributed to delayed treatment. ${ }^{12}$ MRI is a useful diagnostic tool for prompt identification. Following MRI confirmation, if neurological status progressively deteriorates, surgical intervention is warranted. ${ }^{3}$

In the present case, the decision was made to transfer the patient to the operating room, given the substantial neurological deficit observed after the first surgery. Although the argument can be made that imaging prior to returning to the operating room may have prevented an additional surgery, the surgeon felt that expeditious evacuation of the suspected hematoma compressing the spinal cord was warranted to prevent permanent neurological deficits.

Subdural hematomas have been commonly reported in patients with bleeding disorders and patients receiving anticoagulation therapy. ${ }^{1,32}$ In the present report, however, a bleeding disorder was ruled out, and the patient had no history of acute or chronic anticoagulation use. While limited to speculation, the postoperative hypertensive state of the patient raises the question of whether the patient developed the subdural hematoma because of the postoperative hypertension or whether the hypertension occurred as a result of the hematoma compressing the spinal cord.

In the absence of anticoagulation therapy or an underlying coagulopathy, spinal subdural hematomas after spinal surgery are a rare phenomenon. To the best of our knowledge, only 4 reports have described subdural hematoma formation following spinal surgery. ${ }^{5,6,11,13}$ In all reports, hematoma formation was attributed to trauma.

Most recently, in 2013, Gakhar and colleagues described the formation of a subdural hematoma following a left-sided L-5 hemilaminectomy, left L5-S1 facetectomy, and an L5-S1 pedicle screw stabilization and posterolateral fusion. ${ }^{5}$ Postoperatively, the patient developed weakness. An MRI study was ordered, which showed a subdural hematoma extending up to L-1 and a small subdural hematoma at the surgical site. With conservative management, the patient made a full recovery with no residual neurological deficits. The authors specifically noted that the patient was not taking anticoagulants, his preoperative blood tests showed normal results, and there were no visible dural leaks during the procedure..$^{5}$ As such, the authors presumed that the dural manipulation performed during the facetectomy caused the hematoma. ${ }^{5}$

In 2011, Sakai et al. described 2 cases of subdural hematoma formation following lumbar surgery. ${ }^{13}$ Neither patient had intraoperative dural complications or postoperative evidence of cerebrospinal fluid in the drain tubes. In both cases, the symptoms were mild and gradually improved without surgical intervention. The follow-up MR images confirmed resolution of the subdural changes. The authors presumed that the subdural or intradural vessels were compromised during the surgical procedure with resultant hematoma formation..$^{13}$

In the case described by Gehri et al. a spinal subdural hematoma formed following a microdiscectomy and unintended durotomy. ${ }^{6}$ Despite repair, a subdural hematoma subsequently formed at the durotomy site, requiring surgical evacuation. ${ }^{6}$ The patient had notable improvements, but she reported mild back pain and decreased sensation. Lastly, in 1993, Reinsel and colleagues described the formation of a subdural hematoma and credited its formation to trauma during preoperative myelopathy. ${ }^{11}$

In all previous reports of subdural hematoma formation following spinal surgery, the surgical location and formation of the subdural hematoma were in close proximity, ${ }^{5,6,13}$ presumably suggesting intraoperative trauma to the dural tissue. In the present case, however, the subdural hematoma formed in the thoracic spine following a cervical discectomy and fusion, which is suggestive of a different etiology and/or pathomechanism.

Given the spatial discrepancy between the present report and previous reports, the literature was reviewed for cases of remote extradural hematoma formation. Most cases occurred as a complication of intracranial surgery., ${ }^{2,9,15}$ Formation of intracranial hemorrhage has been attributed to multiple factors, including lowered cerebrospinal fluid pressure due to excessive drainage, brain shifting due to imbalanced intracranial pressures, mechanical distension of the dura, and bleeding disorders. ${ }^{2,9,15}$ The pathomechanism responsible for the formation of the subdural hematoma in the thoracic spine remains unclear. It is plausible that gravity and the postoperative hypertension promoted the propulsion of blood from the cervical surgical site into the spinal canal. ${ }^{7}$

To our knowledge, this is the first account of a spinal subdural hematoma in the thoracic spine following surgery at the cervical level. Spinal subdural hematomas should be considered as part of the differential diagnosis in patients demonstrating paraplegia following spinal surgery. With prompt treatment, favorable outcomes can be achieved.

\section{References}

1. Badge R, Chan D: Spinal subdural haematoma in association with anticoagulant therapy, an unusual presentation: a case report and review of literature. Cases J 2:151, 2009

2. Bae KJ, Kim IM, Yim MB: Remote epidural hematoma following the removal of a brain tumor: report of three cases. $\mathbf{J}$ Korean Neurosurg Soc 30:366-370, 2001

3. Domenicucci M, Ramieri A, Ciappetta P, Delfini R: Nontraumatic acute spinal subdural hematoma: report of five cases and review of the literature. J Neurosurg 91 (1 Suppl):65-73, 1999

4. Edelson RN, Chernik NL, Posner JB: Spinal subdural hematomas complicating lumbar puncture. Arch Neurol 31:134137,1974

5. Gakhar H, Bommireddy R, Klezl Z, Calthorpe D: Spinal subdural hematoma as a complication of spinal surgery: can it happen without dural tear? Eur Spine J 22 (Suppl 3):S346-S349, 2013

6. Gehri R, Zanetti M, Boos N: Subacute subdural haematoma complicating lumbar microdiscectomy. J Bone Joint Surg Br 82:1042-1045, 2000 
7. Hicdonmez T, Kilincer C, Hamamcioglu MK, Cobanoglu S: Paraplegia due to spinal subdural hematoma as a complication of posterior fossa surgery: Case report and review of the literature. Clin Neurol Neurosurg 108:590-594, 2006

8. Lee JI, Hong SC, Shin HJ, Eoh W, Byun HS, Kim JH: Traumatic spinal subdural hematoma: rapid resolution after repeated lumbar spinal puncture and drainage. J Trauma 40:654-655, 1996

9. Lim JW, Yang SH, Lee JS, Song SH: Multiple remote epidural hematomas following pineal gland tumor resection. J Pediatr Neurosci 5:79-81, 2010

10. Park J, Ahn R, Son D, Kang B, Yang D: Acute spinal subdural hematoma with hemiplegia after acupuncture: a case report and review of the literature. Spine J 13:e59-e63, 2013

11. Reinsel TE, Goldberg E, Granato DB, Wilkinson S, Penn R: Spinal subdural hematoma: a rare cause of recurrent postoperative radiculopathy. J Spinal Disord 6:62-67, 1993

12. Russell NA, Benoit BG: Spinal subdural hematoma. A review. Surg Neurol 20:133-137, 1983

13. Sakai T, Sairyo K, Bhatia NN, Miyagi R, Tamura T, Katoh S, et al: MRI changes of the spinal subdural space after lumbar spine surgeries: report of two cases. Asian Spine J 5:262266, 2011

14. Singh DK, Chauhan M, Gupta V, Chopra S, Bagaria HR: Spinal subdural hematoma: a rare complication of spinal anesthesia: a case report. Turk Neurosurg 18:324-326, 2008
15. Yacubian EM, de Andrade MM, Jorge CL, Valério RM: Cerebellar hemorrhage after supratentorial surgery for treatment of epilepsy: report of three cases. Neurosurgery 45:159-162, 1999

\section{Disclosure}

The authors report no conflict of interest concerning the materials or methods used in this study or the findings specified in this paper.

\section{Author Contributions}

Conception and design: Wagener. Acquisition of data: Protzman. Analysis and interpretation of data: Wagener. Drafting the article: Protzman, Kapun. Critically revising the article: all authors. Reviewed submitted version of manuscript: all authors. Administrative/technical/material support: Protzman. Study supervision: Wagener.

\section{Correspondence}

Nicole M. Protzman, 3435 Winchester Rd., Allentown, PA 18104. email: nprotzman@coordinatedhealth.com. 